\title{
UCRL-CONF-208523
}

LAWRENCE LIVERMORE N A T IO N A L LABORATORY

\section{Vessel Segmentation and Blood Flow Simulation Using Level-Sets and Embedded Boundary Methods}

T. Deschamps, P. Schwartz, D. Trebotich, P. Colella, D. Saloner, R. Malladi

December 10, 2004

Computer Assisted Radiology Society Conference Chicago, IL, United States

June 23, 2004 through June 26, 2004 
This document was prepared as an account of work sponsored by an agency of the United States Government. Neither the United States Government nor the University of California nor any of their employees, makes any warranty, express or implied, or assumes any legal liability or responsibility for the accuracy, completeness, or usefulness of any information, apparatus, product, or process disclosed, or represents that its use would not infringe privately owned rights. Reference herein to any specific commercial product, process, or service by trade name, trademark, manufacturer, or otherwise, does not necessarily constitute or imply its endorsement, recommendation, or favoring by the United States Government or the University of California. The views and opinions of authors expressed herein do not necessarily state or reflect those of the United States Government or the University of California, and shall not be used for advertising or product endorsement purposes. 


\title{
Vessel Segmentation and Blood Flow Simulation Using Level-Sets and Embedded Boundary Methods
}

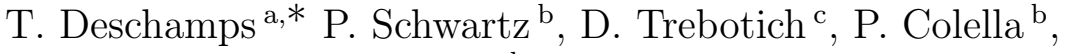 \\ D. Saloner ${ }^{\mathrm{d}}$, R. Malladi ${ }^{\mathrm{a}}$ \\ a Mathematics Department, Lawrence Berkeley National Laboratory \\ ${ }^{\mathrm{b}}$ Applied Numerical Algorithms Group, Lawrence Berkeley National Laboratory \\ ${ }^{\mathrm{c}}$ Center for Applied Scientific Computing, Lawrence Livermore National Laboratory \\ ${ }^{\mathrm{d}}$ Vascular Imaging Research Center, University of California, San Francisco
}

\begin{abstract}
In this article we address the problem of blood flow simulation in realistic vascular objects. The anatomical surfaces are extracted by means of Level-Sets methods that accurately model the complex and varying surfaces of pathological objects such as aneurysms and stenoses. The surfaces obtained are defined at the sub-pixel level where they intersect the Cartesian grid of the image domain. It is therefore straightforward to construct embedded boundary representations of these objects on the same grid, for which recent work has enabled discretization of the Navier-Stokes equations for incompressible fluids. While most classical techniques require construction of a structured mesh that approximates the surface in order to extrapolate a 3D finite-element gridding of the whole volume, our method directly simulates the blood-flow inside the extracted surface without losing any complicated details and without building additional grids.
\end{abstract}

Key words: Blood-Flow, Navier-Stokes equations, Embedded Boundary Methods, Segmentation, Level-Sets, Fast-Marching

* Address: MS50A-1148, Lawrence Berkeley Lab, 1 Cyclotron Rd, Berkeley CA 94709, USA

Preprint submitted to Elsevier Science

15 March 2004 


\section{Introduction}

Computational Fluid Dynamics (CFD) simulations of complex flows in vascular passages such as cerebral or carotid arteries can provide clinicians with information needed to evaluate how pathologies form, how they evolve, and ultimately how they are effectively treated. In order to do this, accurate methods are required to build the anatomical models, and CFD codes must be available that can run on anatomical models such as the carotid artery shown in figure 1 .

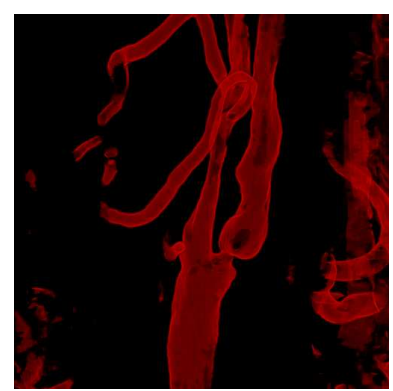

Fig. 1. Volume Rendering of a Carotid artery from a 3D MR dataset

However, methods of surface extraction are limited in their ability to recover the geometry of complex objects like tubular branching structures in real time. Moreover, it is necessary to convert the surface extracted by image processing techniques into a computational domain appropriate for the CFD solver, involving the construction of a mesh on the surface and in the 3D domain surrounding it. Valuable information can be lost during this construction due to limitations of the CFD solvers with respect to the properties of the surface mesh. It is often the case, for example, that the surface needs to be smoothed in order to build a finite-element mesh necessary to some CFD codes. Failing that, irregular surfaces must be approximated by a large number of small mesh elements, pushing the limits of computer memory. The result in this and other cases is a compromise in the level of accuracy of the surface extraction method.

Some recent techniques of image processing enable an approximation of the surface of any vascular tree in the patient body to be built using CT or MR images of the patient enhanced with contrast product. This work is based on an accurate surface extraction with front propagation techniques based on the Fast-Marching and Level-Sets methods [1].

In addition, recent techniques in CFD make easy use of these approximate surfaces in the generation of computational grids: a higher-order projection method has been desgined on Chombo, a software framework for applied partial differential equation (PDE) solvers on irregular domains.

The purpose of this work, therefore, is to transform the result of an accurate surface extraction method detailed in section 2 into a CFD mesh detailed in section 3 with no loss of information. Using a high-resolution CFD code presented in section 4, 
we accurately compute measures of fluid velocity, pressure and wall shear stress in realistic arterial geometries.

\section{Surface Extraction}

Fast-Marching and Level-Sets methods are numerical techniques which can follow the evolution of contours and surfaces that can develop sharp corners, break apart, and merge together, and are particularly useful for shape recovery of complex geometries like branching tubular structures [2].

Rather than tracking the movement of a given contour $C$ moving with speed $F$ in its normal direction $\vec{n}$ according to the evolution equation $\frac{\partial C}{\partial t}=F \vec{n}$, we consider the signed distance function $\phi$ to $C$ and track its motion with the evolution equation

$$
\frac{\partial \phi}{\partial t}=F|\nabla \phi|, \text { with } F=k_{I}(1-\epsilon \kappa)-\nabla k_{I} \cdot \vec{n}, \text { and } k_{I}(\mathbf{x})=e^{-\|\nabla I \mathbf{x}\|}
$$

This is the Level-Sets method [1]. Therefore since the contour is moved implicitly by the evolution of the signed distance function, the resulting surface extraction exhibits good properties including a total adaptability to complex topologies like branching arterial structures and a sub-pixel accuracy of the surface thanks to the implicit formulation of the sign distance function. An example is shown in figure 2 When the speed $F$ is strictly positive and a function of the image only, equation 1 re-
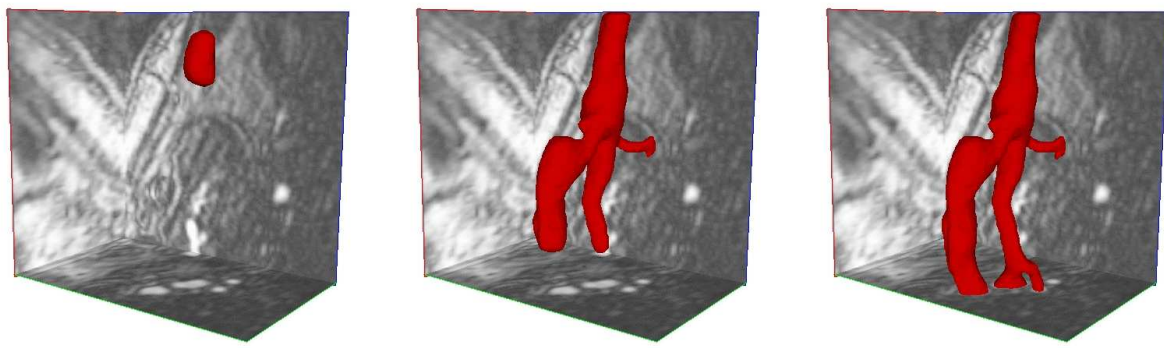

Fig. 2. Carotid segmentation with Level-Sets: the propagating front at three successive times.

solves in the stationary case $F|\nabla T|=1$, with $F(\mathbf{x})=k_{I}(\mathbf{x})$ defined in eq (1)where $T$ is the crossing time of the propagating front. Since Level-Sets are rather slow, initialization of the segmentation can be done with the Fast-Marching method [1] that solves Eikonal equation in $o(n \log (n))$ (where $n$ is the number of points in the image). When coupled [3], these numerical techniques can achieve the highest level of accuracy in a very short time, compared to other segmentation techniques like classical active contours that fail to capture complex shapes. These algorithms have been validated in numerous cases [4], and can be specifically optimized to extract long and thin tubular objects [5]. 


\section{Building the CFD grid}

In order to simulate the blood-flow inside the carotid that we just segment, many methods rely on a finite element griding of the 3D domain that is based on remeshing appropriately the surface extracted. Re-meshing often discards a lot of the surface details because it is done with approximation of the surface (like NURBS) and involves a lot of user-interaction. Other methods that want to keep all the details end up with quantity of mesh elements (like triangles) that are challenging both for computers' memory and commercial CFD softwares.

The embedded boundary method is an approach in which regular cells on a Cartesian grid are cut by physical boundaries and/or interfaces (see Figure 3). Higher-order stencils are constructed near boundaries where irregular, or "cut", cells occur while discretizations with known accuracy and stability requirements are employed on the interior regular cells $[6,7]$.

The geometric facts required for numerical methods (normals, centroids, face areas, and so forth) can each be realized as integrals of monomials over the volumeof-fluid. There is in general more than one such integral for each geometric datum. Hence the set of all such integrals constitutes an overdetermined system for the geometry. We apply Least Squares to this over-determined system, which finesses some difficult aspects of computational geometry. Furthermore, by using the divergence theorem we are able to replace volume integrals over irregular regions with trivial computations of one-dimensional integrals over line-segments.

Given a level set description of the EB as above, grid generation proceeds as an automated process taking a few minutes (see figure 3 ).
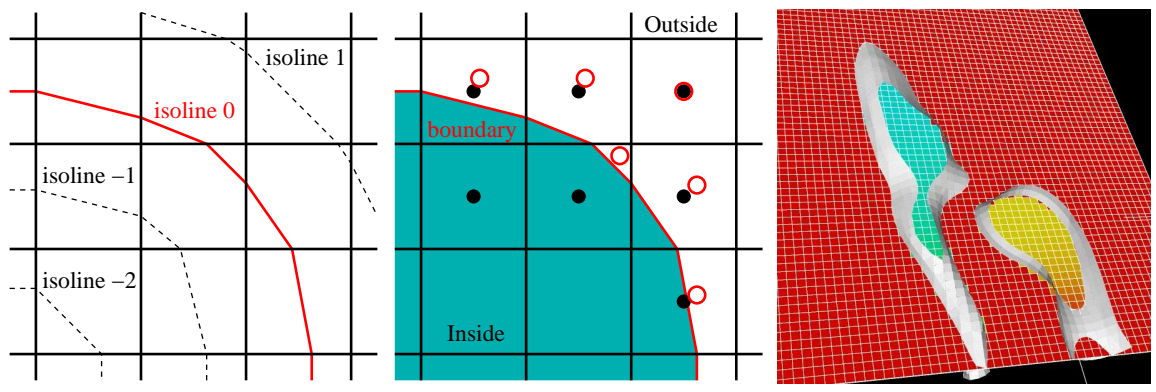

Fig. 3. From Level-Sets to Embedded Boundary - Left image: the distance function on the Cartesian grid - Middle image: the different cells, their centers (black disks) and centroids (empty disks) - Right Image: the Surface and the underlying Cartesian grid for the carotid example.

\section{Blood-Flow Simulation}

We make use of the Chombo software libraries [8] which provide a framework for data management, numerical operators and linear solvers needed in an EB 
formulation. We adapt the Chombo framework to incompressible flow by developing fundamental numerical operators requiring viscous (Dirichlet) boundary conditions.

We model blood flow with the incompressible Navier-Stokes equations which are a combination of evolution equations, and a constraint due to the incompressibility condition:

$$
\rho u_{t}+\rho(u \cdot \nabla) u=-\nabla p+\mu_{s} \Delta u \text {, and } \nabla \cdot u=0 .
$$

The formulation of appropriate time-discretization methods is more subtle than that for evolution equations alone. To address this issue, Chorin [9] introduced projection methods based on the Hodge decomposition of any vector field into a divergencefree part and the gradient of a scalar field. Projection methods are fractional step methods for which an intermediate velocity is computed that does not necessarily satisfy the incompressibility constraint. This velocity is then corrected so that it satisfies the constraint. In [10] was introduced a predictor-corrector method based on Chorin's ideas. Some of the key advantages of their method are that the advective terms can be treated using explicit high-resolution finite difference methods for hyperbolic PDEs, and that the only linear systems to be solved are ones resulting from standard discretizations of second-order elliptic and parabolic PDEs which are amenable to solution using fast iterative methods such as multigrid. This leads to a method that is second-order accurate in space and time. It has a stability constraint on the time step due only to the CFL condition for the advection terms, and a robust treatment of underresolved gradients in the Euler limit.

We present in figure 4 results for the flow simulated in the stenotic carotid artery of figure 1. Fluid velocity and pressure are computed with the flow solver and visualized on a desktop workstation. Pressure drops (brighter areas) and complex flow patterns are observed near the arterial bifurcation and beyond the stenosis.

\section{Conclusion}

Our approach to computing flows in realistic arterial geometries with stenosis does not modify the object geometry from the imaging, and uses a fast, automated process for generating an appropriate finite volume grid. We have a direct construction of the embedded boundary on the Cartesian grid, and thus no loss of information nor accuracy. The final flow simulation results are obtained for the realistic geometry that has been extracted by the segmentation method. Future work will concern the use of adaptive mesh refinement techniques and parallel implementation to reduce the computing cost of the CFD simulation, as well as more complex simulations built on the Chombo library involving fluid-surface interactions and influence of other materials like plaque and stents. 


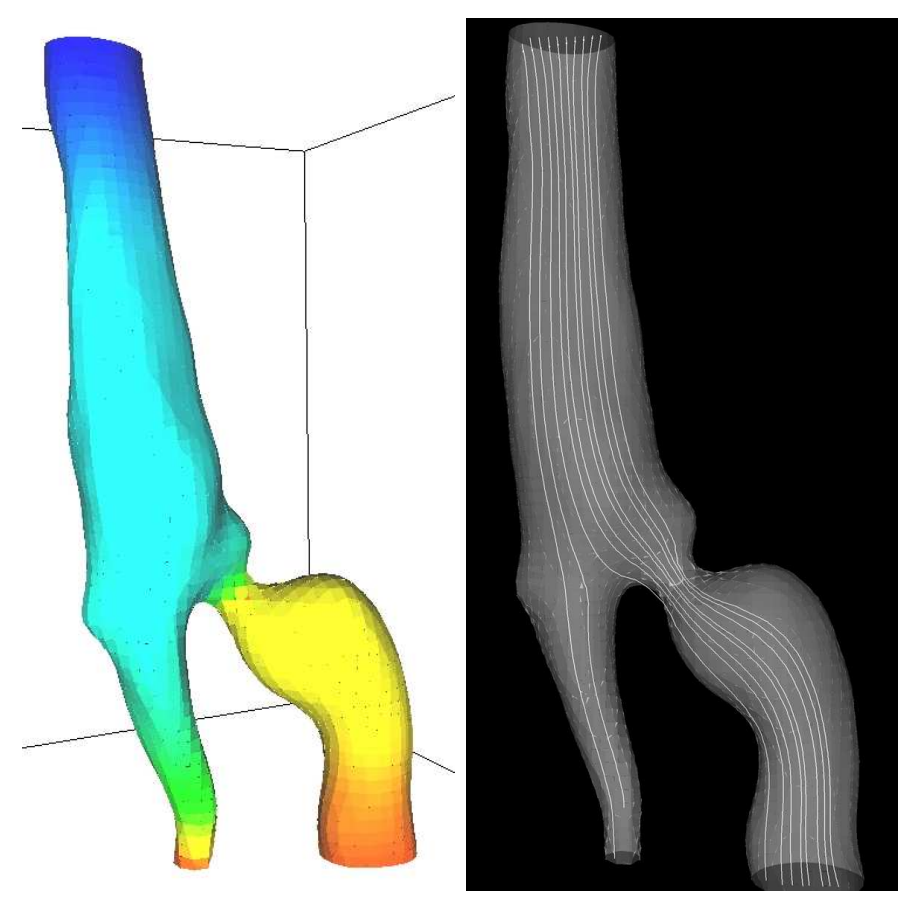

Fig. 4. Blood-Flow simulation inside the carotid - Left image: Pressure on the surface; Right image: iso-surfaces of the longitudinal component of the flow velocity.

\section{References}

[1] J. Sethian, Level set methods: Evolving Interfaces in Geometry, Fluid Mechanics, Computer Vision and Materials Sciences, Cambridge University Press, UC. Berkeley, 1999.

[2] R. Malladi, J. Sethian, B. Vemuri, Shape modelling with front propagation: A level set approach, IEEE Trans. Pattern Analysis And Machine Intelligence 17 (2) (1995) 158-175.

[3] R. Malladi, J. Sethian, A real-time algorithm for medical shape recovery, 1998, pp. 304-310.

[4] M. Hernandeza, R. Barrenab, G. Hernandezb, G. Sapiroc, A. Frangi, Pre-clinical evaluation of implicit deformable models for three-dimensional segmentation of brain aneurysms in cta, in: SPIE Medical Imaging, Vol. 5032, 2003, pp. 1264-1274.

[5] T. Deschamps, L. Cohen, Fast surface and tree structure extraction of vascular objects in 3d medical images, in: Intl. Conf. on Curves and Surfaces, Saint-Malo, France, 2002.

[6] H. Johansen, P. Colella, A cartesian grid embedded boundary method for poisson's equation on irregular domains, J. Comput. Phys. 147 (2) (1998) 60-85.

[7] P. McCorquodale, P. Colella, H. Johansen, A cartesian grid embedded boundary method for the heat equation on irregular domains, J. Comput. Phys. 173 (2001) 620-635.

[8] P. Colella, D. Graves, T. Ligocki, D. Martin, D. Modiano, D. Serafini, B. V. Straalen, Chombo software package for amr applications (2000).

[9] A. Chorin, Numerical solutions of the navier-stokes equations, Math. Comp. 22.

[10] J. Bell, P. Colella, H. Glaz, A second-order projection method for the incompressible navierstokes equations, J. Comput. Phys. 85 (1989) 257-283. 